\title{
Reizdarmsyndrom und Ernährung: Reizende Diagnose und Therapie!
}

\author{
Christiane Schäfer
}

Mit Veröffentlichung der ROME-IV-

Kriterien für die Diagnose des Reizdarmsyndroms (RDS) wächst die bittere

Erkenntnis, dass einfache Lösungen bei der Diagnostik und Therapie dieses

Krankheitsbilds unmöglich sind. Neben immer komplizierteren Diagnose-

algorithmen, die zum Ausschluss anderer pathologischer Zustände abgearbeitet werden müssen, besteht bei den

Therapieoptionen Bedarf für eine bessere interdisziplinäre Zusammenarbeit.

Trotz großer klinischer Bedeutung werden die neurogastroenterologischen Erkrankungen, zu denen das RDS gehört, von Ärzten und Ernährungstherapeuten aufgrund unzureichender Kenntnis über den aktuellen Wissensstand häufig nicht als organische Erkrankung anerkannt, sondern als eingebildetes Beschwerdebild oder Befindlichkeitsstörung interpretiert $[8,9,11,27,42]$.

\section{Prävalenz und \\ Ursachenforschung}

Bis zu 30\% der Patienten stellen sich heute in einer Arztpraxis mit zunächst unklar zu fassenden gastrointestinalen Symptomen vor. Sieben bis zwölf Prozent der Patienten lassen sich der Diagnose Reizdarmsyndrom zuordnen. Die Lebensqualität der Betroffenen kann spürbar eingeschränkt sein. Frauen sind etwas häufiger betroffen als Männer und die Symptome kommen in jedem Lebensalter vor $[14,15,32]$.

Zunehmend bestimmen Erkenntnisse der Neurogastroenterologie das Geschehen. Der bis Mai 2016 verwendete Begriff „funktionelle Störungen“ (FGID: Functional Gastrointestinal Disorders) hat in anderssprachigen Ländern für Irritationen

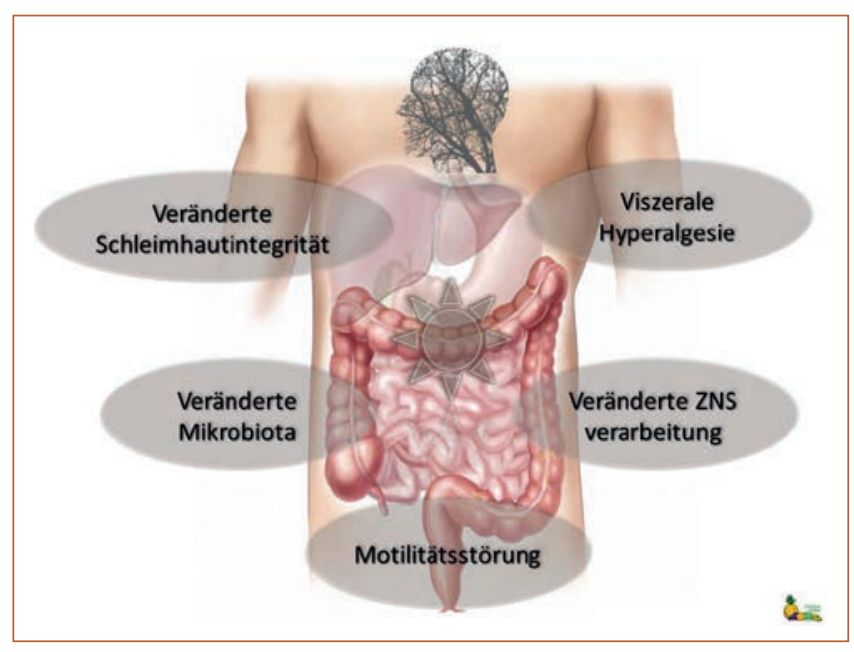

Abb. 1 Hypothese: RDS als Störungen der Darm-Hirn-Interaktion.

in dem ohnehin schwer zu durchschauenden RDS-Diagnosepfad gesorgt. Da sich der Begriff nicht treffend übersetzen ließ, wurde eine neue Begrifflichkeit eingeführt: Störungen der Darm-Hirn-Interaktion (DGBI: Disorders of Gut-Brain Interaction). Eine Vielzahl neuer diagnostischer Kriterien ermöglicht zudem die Identifikation anderer Erkrankungen und damit eine Abgrenzung zum RDS. Die Kenntnis um andere pathologische Ursachen (u.a. Erkrankungen des Ösophagus, gastroduodenale Störungen, Gallensäuretransportstörungen, anorektale Störungen) ist Voraussetzung zur Sicherung der Diagnose und Therapie eines RDS $[43,47]$.

\section{RDS - das Diagnoseproblem}

Die Diagnose „Reizdarmsyndrom“ wird erst gestellt, wenn vorangegangene Untersuchungen zweifelsfrei belegen, dass keine andere, die Symptome begründende Erkrankung vorliegt. Das RDS bleibt ein heterogenes Krankheitsbild, bei dem verschiedene Mechanismen allein oder aber in Kombination zu Beschwerden führen $(\triangleright$ Abb. 1$)$.
Inwieweit eine Dysbiose eine veränderte intestinale Permeabilität und daraus resultierend eine veränderte Immunantwort generiert, ist Gegenstand aktueller Forschung. Daten aus Studien zum Mikrobiom deuten an, dass der Verlust von Diversität die Zellintegrität des Gastrointestinaltrakts schwächt. Aktuelle, von der Erkrankung unabhängige Forschungen stellen die Förderung und (Wieder-) Herstellung einer möglichst breiten Diversität für eine effiziente Therapie des RDS in den Fokus $[33,45]$.

Ein anderer wesentlicher Mechanismus wird in der Hypersensitivität des Darmtrakts gegenüber chemischen und mechanischen Reizen vermutet. Da die Interaktion bidirektional verläuft (HirnDarm-Achse) ist hier unklar, ob die veränderte Immunantwort Folge oder Ursprung der Erkrankung ist. Die Identifikation dieser viszeralen Hypersensitivität und Hinweise zu vorliegenden Motilitätsstörungen fließen in die Klassifikation der RDS-Therapie der unterschiedlichen Subtypen ein $[4,7,8]$.

RDS wird weiterhin als Symptomenkomplex definiert, bei dem länger als 
drei Monate anhaltende oder wiederkehrende Symptome auftreten. Da die Bewertung der Symptome subjektiv ist, können sie nur in wenigen Fällen objektiviert werden. Kennzeichnend bleiben Stuhlunregelmäßigkeiten wie Diarrhö und/oder Obstipation, die auch im Wechsel auftreten können, ebenso wie Schmerzen oder Druckgefühl im Bauch sowie Blähungen [11]. In der Zusammenschau mit Angaben zur Stuhlkonsistenz und -frequenz werden Therapieoptionen abgeleitet [11, 29, 43].

Über Monate anhaltende Beschwerden lassen Patienten immer wieder nach Ursachen fahnden. Klare pathologische oder histologische Veränderungen, die zur Diagnose herangezogen werden könnten, fehlen. Dementsprechend gibt es auch kein morphologisches Korrelat, das diese Beschwerden erklärt. Wechselnde Therapeuten, viele - zum Teil sich deutlich unterscheidende - Arztberichte und leidvolle Erfahrungen pflastern den Diagnoseweg. Vielfältige Symptome und manchmal gravierende Einschränkungen der Lebensqualität bedürfen verschiedenster inhaltlicher und methodischer Tools [10, 13, 41] ( Abb. 2).

Die primäre RDS-Diagnostik beruht nach wie vor auf einer Ausschlussdiagnostik. Eine sorgfältig erhobene Anamnese, die Durchführung klinischer Untersuchungen sowie gezielte Funktionsuntersuchungen (Ösophagogastroduodenoskopie, Koloskopie, $\mathrm{H}_{2}$-Atemtest, Ausschluss von Tumoren, Laboruntersuchungen u.v.m.) sind zwingende Voraussetzungen für die Diagnosestel-

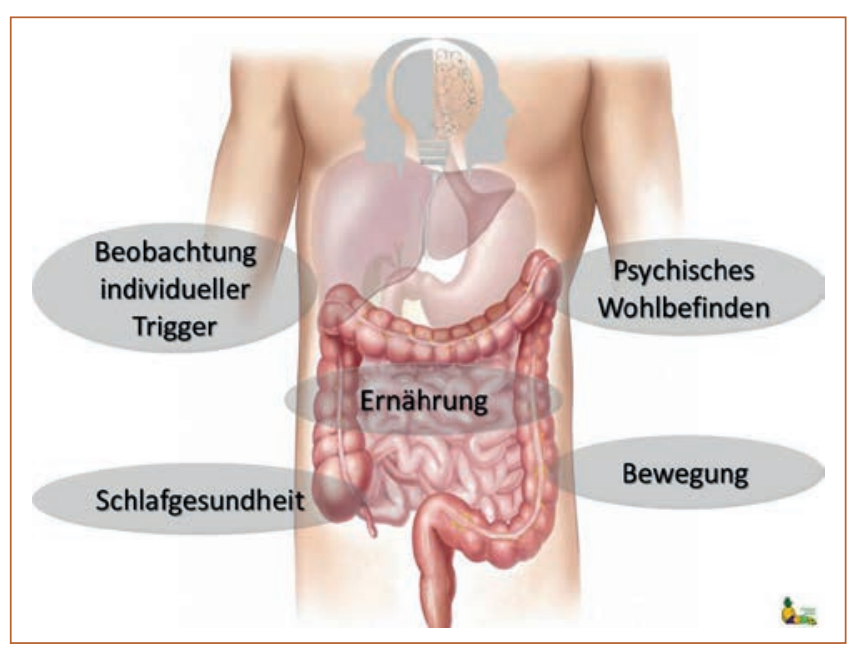

-Abb. 2 Überblick über allgemeine Therapiemaßnahmen bei RDS. lung. Ernährungsfehler, Kohlenhydratmalassimilationen oder organische Ursachen werden noch immer zu regelhaft übersehen [1, 22, 29, 40].

ROME IV unterscheidet statt bisher drei nun vier RDS-Subtypen ( $\triangleright$ Tab. 1 ), trotz der bleibenden drei Symptomenkomplexe (Diarrhö, Obstipation, Flatulenz/Meteorismus). Diese symptombasierte Definition birgt die Gefahr der deutlichen Überlappung mit anderen Krankheitsbildern, die ähnliche Beschwerden zeigen (z.B. Symptomatic Uncomplicated Diverticular Disease [SUDD], funktionelle Dyspepsie, chronische Obstipation).

Tab. 1 Die vier Reizdarm-Typen - nach dem jeweiligen Hauptsymptom (nach [11, 14]).

\begin{tabular}{|l|l|}
\hline Typ & Symptom/Kriterien \\
\hline $\begin{array}{l}\text { RDS-D } \\
\text { (diarrhöbetont) }\end{array}$ & $\begin{array}{l}\text { dieser RDS-Typ zeigt sich mit dem überwiegenden Symptom } \\
\text { Diarrhö bei den Betroffenen. Kriterium: Mehr als ein Viertel (>25\%) } \\
\text { der Stuhlgänge entsprechen der Bristol Stool Scale 6-7 (sehr weich } \\
\text { bis flüssig) und weniger als ein Viertel (<25\%) den Typen 1-2 (hart, } \\
\text { klumpig, schafskotartig) }\end{array}$ \\
\hline $\begin{array}{l}\text { RDS-O } \\
\text { (obstipationsbetont) }\end{array}$ & $\begin{array}{l}\text { überwiegendes Symptom ist die Obstipation. Kriterium: Mehr als } \\
\text { ein Viertel (>25\%) der Stuhlgänge entsprechen der Bristol Stool }\end{array}$ \\
\hline $\begin{array}{l}\text { Scale 1-2 und weniger als ein Viertel (<25\%) den Typen 6-7 } \\
\text { (Mischtyp) }\end{array}$ & $\begin{array}{l}\text { dieser RDS-Typ zeigt sich mit wechselnden Symptomen der Diarrhö } \\
\text { und Obstipation bei den Betroffenen, wobei auch Meteorismus und } \\
\text { Flatulenz auftreten können. Kriterium: Mehr als ein Viertel (>25\%) } \\
\text { der Stuhlgänge entsprechen der Bristol Stool Scale 1-2 und mehr als } \\
\text { ein Viertel (>25\%) den Typen 6-7 }\end{array}$ \\
\hline $\begin{array}{l}\text { RDS-U } \\
\text { (untypisiert) }\end{array}$ & $\begin{array}{l}\text { vielfältige, wechselhafte gastrointestinale Beschwerden, keine } \\
\text { eindeutige Symptomatik. Kriterium: Patienten erfüllen die } \\
\text { diagnostischen Kriterien für ein RDS, aber ihre Darmbeschwerden } \\
\text { können nicht genau genug einem der o.g. Subtypen zugeordnet } \\
\text { werden }\end{array}$ \\
\hline
\end{tabular}

\section{Anspruchsvoll: Ernährungs- therapie beim RDS}

Der oft überbewertete Einfluss der Ernährung birgt die Gefahr der Fehl- und Mangelernährung durch eingeleitete Karenzmaßnahmen. Da die Symptome des RDS durch den Verzehr von Lebensmitteln $\mathrm{zu}$ beeinflussen sind, besteht ein hoher Erfolgsdruck hinsichtlich der Wirksamkeit der verschiedenen ernährungstherapeutischen Interventionen [2, 31, 36]. Aber: Sinnvolle Therapieoptionen, die die Mehrzahl der Patienten auf Dauer zufriedenstellen könnte, gibt es nicht. Denn systematische und haltbare wissenschaftliche Aussagen zu ernährungsspezifischen Therapieoptionen liegen auch nach ROME IV nicht vor. Weiterhin fehlt es an klaren Biomarkern, nach denen sich Ernährungsoptionen richten könnten. Der gestörte Gallensäuremetabolismus zeigt sich zwar bei $40 \%$ der Patienten mit RDS-D, lässt sich mit praxistauglichen Diagnosemethoden aber nicht effizient erfassen. Daher empfiehlt sich weiterhin das symptomorientierte individuelle Arbeiten in der Ernährungstherapie $[16,19,36]$ ( $\triangleright$ Abb. 3, $\triangleright$ Tab. 2).

Die generelle Herausforderung besteht darin, dass das Spektrum der Lebensmittelauswahl und die Relevanz nahrungsmittelbezogener Trigger groß sind und von Patient zu Patient eine starke Variabilität zeigen. Inter- und intra- 


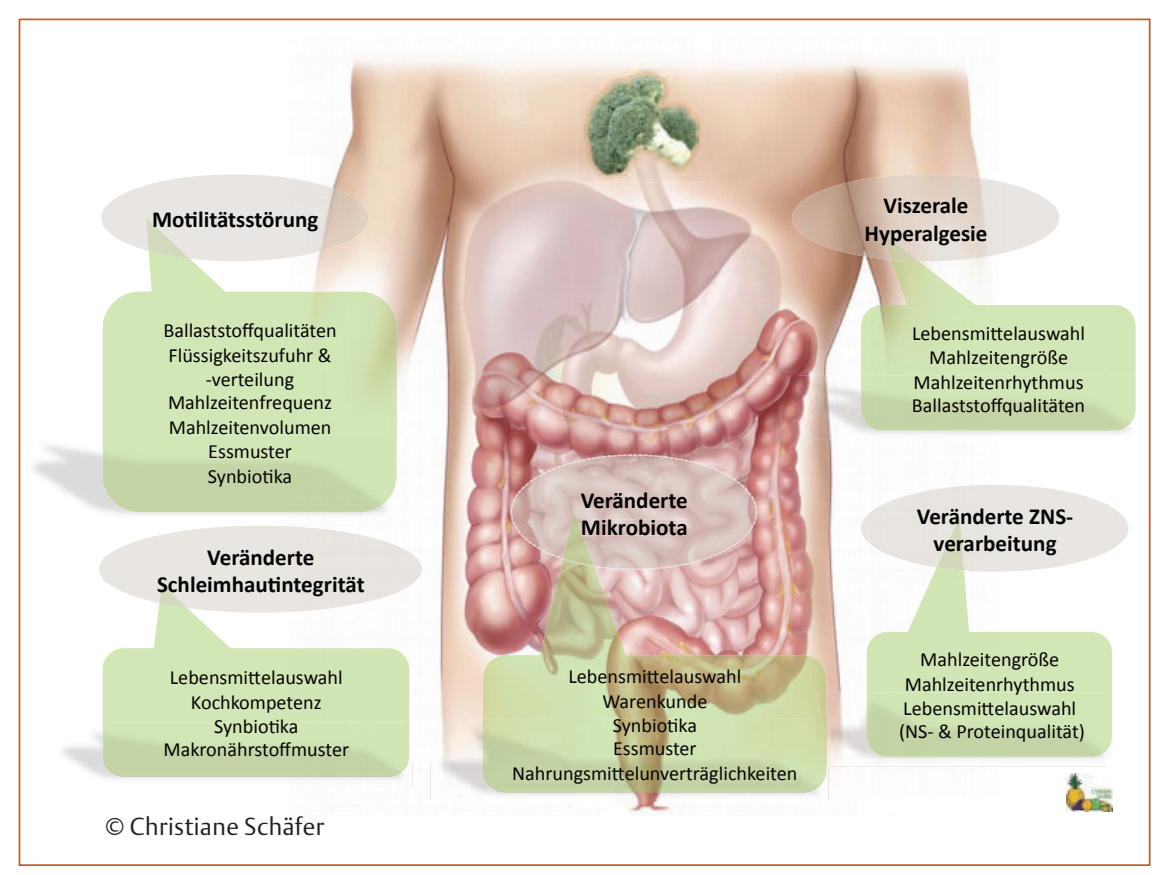

-Abb. 3 Ernährungstherapeutische, symptomorientierte Ansatzpunkte bei RDS. oder ein Votum für eine bestimmte Ballaststoffqualität weder studienmäßig sinnvoll zu erfassen, noch in Kostformen adäquat zu berechnen [3, 20]. Demzufolge ist es nicht überraschend, dass die klinische Wirksamkeit eines Ballaststoffeinsatzes im Allgemeinen insgesamt nicht eindeutig belegt ist [14, 16, 17, 36]. Das darf aber nicht darüber hinwegtäuschen, dass die Veränderung von Ballaststoffqualitäten probatorisch erwogen werden sollte [6, 19, 29, 44].

\section{Einen Versuch wert: Probiotika}

Während bestimmte Ballaststoffe als Präbiotikum das Mikrobiom beeinflussen, ist der gezielte Einsatz von Probiotika Gegenstand zahlreicher systematischer Übersichtsarbeiten. Ähnlich wie bei den Ballaststoffen scheitert eine konkrete Empfehlung jedoch an den hetero- individuelle Unterschiede erschweren die Erklärung pathologischer Zusammenhänge in Abgrenzung von unphysiologischen Ernährungsstrategien. Daher ist es zum jetzigen Zeitpunkt nicht gerechtfertigt, allgemeingültige RDSDiäten und strikte Karenzempfehlungen zu verordnen [2, 14, 21, 42]. Dies ist umso wesentlicher, da die Mikrobiomforschung die protektive Rolle einer hohen Diversität unmissverständlich betont. Protektive Keime, die nachweislich durch präbiotische Substanzen in ihrem Besatz gefördert werden, stehen den pauschalen Karenzempfehlungen entgegen [18, 34].

\section{Das Ballaststoff-Dilemma}

Da Beschwerden im Gastrointestinaltrakt im Zusammenhang mit der Lebensmittelauswahl und -zusammensetzung stehen, liegt es nahe, einzelnen Komponenten der Nahrung einen besonderen therapeutischen Effekt zu unterlegen. Die Sichtweise, dass das RDS auch aus einer gestörten oder veränderten Motilität resultiert und mit einer viszeralen Hyperalgesie einhergeht, hat den Ballaststoffen eine besondere Rolle zugetragen. Doch genau hier liegt das Dilemma: Aufgrund der Inhomogenität der Ballaststoffe ist eine konkrete Zufuhrempfehlung
\Tab. 2 Ziele und Inhalte ernährungstherapeutischer Interventionen bei RDS

\begin{tabular}{|c|c|}
\hline symptomorientierte Ziele & $\begin{array}{l}\text { - regelmäßiger Stuhlgang } \\
\text { - „normale“ Stuhlkonsistenz } \\
\text { - Senkung der Beschwerdeamplitude } \\
\text { - Wiederherstellung der Lebensqualität }\end{array}$ \\
\hline ernährungstherapeutische Ziele & $\begin{array}{l}\text { - Sicherung der Nährstoffbilanzierung } \\
\text { - Erarbeitung physiologischer Ernährungs- } \\
\text { muster nach individuellen Lifestylevorgaben } \\
\text { und Kochkompetenz } \\
\text { - Vermeidung unsinniger (zu langer) Karenz- } \\
\text { maßnahmen } \\
\text { - möglichst einfache, gut in den Alltag } \\
\text { transferierbare Empfehlungen }\end{array}$ \\
\hline $\begin{array}{l}\text { sinnvolle spezielle Nahrungsmittel- } \\
\text { interventionen }\end{array}$ & $\begin{array}{l}\text { - Ballaststoffzufuhr qualitativ überprüfen und } \\
\text { ggf. austauschen, senken oder z. T. auch } \\
\text { erhöhen } \\
\text { - Verzehr von Sauermilchprodukten und } \\
\text { Synbiotika überprüfen und ggf. in den } \\
\text { Speiseplan integrieren } \\
\text { - Protein-/Lipidgehalt der Nahrung überprüfen } \\
\text { und ggf. anpassen } \\
\text { - Flüssigkeitszufuhr und deren Verteilung } \\
\text { überprüfen } \\
\text { - Einsatz von Pro- und Präbiotika abwägen }\end{array}$ \\
\hline Ernährungsmuster & $\begin{array}{l}\text { - Mahlzeitenabstände kontrollieren und } \\
\text { individuelle Muster herausarbeiten (insbeson- } \\
\text { dere bei Patienten mit Meteorismus) } \\
\text { - Mahlzeitenkomposition kontrollieren, ggf. } \\
\text { anpassen }\end{array}$ \\
\hline Hilfsmittel & $\begin{array}{l}\text { - medizinische Vorbefunde, insb. Labor } \\
\text { - Ernährungs- und Symptomprotokoll } \\
\text { - Stuhlprotokoll } \\
\text { - Beschwerdebarometer } \\
\text { - Wohlfühlkalender }\end{array}$ \\
\hline
\end{tabular}


genen, stammspezifischen Eigenschaften der Probiotika, die zusätzlich eine Abhängigkeit von Ernährungsmustern zeigen [6, 25, 26, 36, 37]. Gleichwohl rechtfertigen die Wirkungsbreite und das therapeutische Potenzial einzelner Stämme - v.a. aus der Gattung der Bifidobakterien - den symptomorientierten Einsatz.

\section{Low FODMAP: ja oder nein?}

In der Praxis wird zudem mit den unterschiedlichsten Low-FODMAP-Diäten ein therapeutischer Einsatz entsprechender Kostformen erwogen. Eine Metaanalyse zum Einsatz von Low-FODMAP-Diäten bei Patienten mit RDS zeigt zwar die Senkung des Symptomscores hinsichtlich abdomineller Beschwerden bei einer Reduktion des Anteils an FODMAPs; die mangelnde Vergleichbarkeit der untersuchten Low-FODMAP-Kostformen erschwert jedoch die Formulierung genereller Aussagen zu deren Wirksamkeit [6, 23, 25, 46]. Am Beispiel der Fruktane werden Kontroversen deutlich: Zum einen formulieren die Studien den sinnvollen präbiotischen Einsatz zur Steigerung der protektiven Diversität, zum anderen wird eine symptomauslösende Wirkung (Dysbiose) diskutiert [6, 24, 39]. Eine pauschale Karenzempfehlung kann somit nicht abgeleitet werden. Vielmehr wird eine individuelle Anpassung der Kostformen an die fermentierbaren Kohlenhydrate gefordert [12]. Eine individuelle Anpassung stellt die Grundvoraussetzung bei Anwendung von LowFODMAP-Diätversuchen dar, da die langfristige Einhaltung einer strengen Low-FODMAP-Diät zu ernährungsbedingten Nährstoffmangelzuständen und zu negativen Auswirkungen auf das Mikrobiom führt [26]. Die langfristige Wirksamkeit dieser Diätform sowie der Kostaufbau nach der diätetischen Karenz sind Gegenstand weiterer Studien [31, 34, 35, 36].

Da Betroffene jegliche Nahrungsaufnahme als Auslöser von Symptomen verantwortlich machen, sind sie für strikte Karenzdiäten und Fehlernährungstendenzen empfänglich. Diese sind zu vermeiden, zumal komplexe Ernährungsstrategien von der Mehrheit der Patienten oft nicht eingehalten werden [22]. Pauschalempfehlungen bzw. ein einheit- liches therapeutisches Vorgehen wäre für diese äußerst heterogene Patientenklientel mit komplexem Beschwerdebild weder angemessen noch effektiv [5, 22, 48]. Dies gilt vor allem für den Verzicht auf Grundnahrungsmittel (z.B. milchoder glutenfreie Kostvarianten), da diese nachweislich negative Effekte zeigen [30]. Wenn diese Mahlzeitengestaltung ohne Kochkompetenz seitens der Patienten in den Alltag transferiert werden muss, ist die Kenntnis der Warenkunde unbedingt erforderlich [47].

Gerade weil eine „Heilung“ im ursprünglichen Wortsinn nicht möglich ist, geht es in der Ernährungstherapie eher darum, dogmatische Vorgaben zu verhindern. Dafür sollten individuelle Vorlieben am täglichen Essen und der Mahlzeitengestaltung herausgearbeitet werden. Nur so kann dem Faktor Ernährung der ihm eigene Stellenwert zugeordnet werden. Die häufig erwartete konkrete Angabe, welche Nahrungsmittel ohne Probleme verzehrt werden können, bleibt jede Beraterin schuldig.

Die Basis der Ernährungstherapie umfasst vor allem die Aufklärung des Patienten hinsichtlich der Ungefährlichkeit der vorliegenden Symptome. Aufgrund möglicher Einschränkung der Lebensqualität sollte diesem Therapieteil größtmögliche Sorgfalt gewidmet werden [15, 28, 37].

\section{Ernährungstherapie ist nicht alles!}

Das Wissen um Pathogenese, Medikamente und psychologische Feinheiten ist für alle Beteiligten unerlässlich und verdeutlicht, dass dieses Krankheitsbild, wenn überhaupt - auch aus ernährungstherapeutischer Sicht - nur im interdisziplinären Team zu begleiten ist. Die Vielzahl möglicher Behandlungsoptionen reflektiert die Unsicherheiten und auch das Unwissen bezüglich der verschiedenen derzeit diskutierten pathophysiologischen Mechanismen.

Um die Eigenverantwortung des $\mathrm{Pa}$ tienten zu stärken, sollten folgende Inhalte in einer gastroenterologischen Ernährungstherapie bearbeitet werden:

- Den Patienten unterstützen und ihm versichern, dass die Beschwerden real und nicht eingebildet sind (Einsatz eines Beschwerdebarometers/ Symptomprotokolls).

- Dem Patienten verdeutlichen, dass das Krankheitsbild dauerhaft vorhanden ist, aber hinsichtlich der Beschwerdesymptomatik in wechselnden Intensitäten verlaufen kann.

- Herausarbeiten von individuellen, durchaus zahlreich möglichen Triggerfaktoren, die Beschwerden auslösen können, z. B.

- große Mahlzeiten, Essmuster u.a.

- Stress, Medikamente und andere Irritanzien (scharfe Gewürze, Alkohol).

- Beeinflussbarkeit der Beschwerden durch psychische Faktoren (Bauchhirn, „brain-gut-axis“) thematisieren. Darmbezogene Hypnotherapie kann ergänzend sinnvoll sein [38].

- Wiederholtes Verdeutlichen, dass keine Lebensbedrohung und keine Krebsgefahr besteht.

- Hinweis auf die Unsinnigkeit wiederholter diagnostischer Untersuchungen sowie darauf, dass ein Ärzte- oder Therapeutenhopping nicht zielführend im Sinne des Krankheitsmanagements ist.

Derzeit erzielt die Ernährungstherapie bei Patienten mit RDS die besten Erfolge, wenn sie eine individualisierte und symptombezogene Behandlungsstrategie verfolgt. Nur so kann die Fülle an Beschwerden beherrscht und dem Patienten ein Versorgungsalgorithmus angeboten werden, ohne ausufernde und stets neue Behandlungsmöglichkeiten zu offerieren $[16,28,48]$.

Online

https://doi.org/10.1055/s-0043-118118

\section{Literatur}

1 Andresen V, Keller J, Pehl C et al. Irritable bowel syndrome - the main recommendations. Dtsch Arztebl Int 2011; 108: 751-760

2 Barbara G, Feinle-Bisset C, Ghoshal U et al. Intestinal microenvironment and functional gastrointestinal disorders. Gastroenterology 2016; 150: 1305-1318

3 Becker SR, Weber MA. Ballaststoffe. Schweiz Zschr GanzheitsMedizin 2002; 1: 32-40 
4 Boeckxstaens G, Camilleri M, Sifrim D et al. Fundamentals of neurogastroenterology: physiology/motility - sensation. Gastroenterology 2016; 150: 1292-1304

5 Bohn L, Storsrud S, Liljebo T et al. Diet low in FODMAPs reduces symptoms of irritable bowel syndrome as well as traditional dietary advice: A randomized controlled trial. Gastroenterology 2015; 149: 1399-1407

6 Brouns F, Delzenne N, Gibson G. The dietary fibers-FODMAPs controversy. Cereal Foods World 2017; 62: 98-103

7 Buhner S, Qin L, Vignali S et al. Activation of human enteric neurons by supernatants of colonic biopsy specimens from patients with irritable bowel syndrome. Gastroenterology 2009; 137: 1425-1434

8 Camilleri M, Boeckxstaens G. Dietary and pharmacological treatment of abdominal pain in IBS. Gut 2017; 66: 966-974

9 Camilleri M, Buéno L, Andresen V et al. Pharmacologic, pharmacokinetic, and pharmacogenomic aspects of functional gastrointestinal disorders. Gastroenterology 2016: 150: 1319-1331

10 Chey W, Whelan K. Dietary guidelines for irritable bowel syndrome are important for gastroenterologists, dietitians and people with irritable bowel syndrome. J Human Nutr Diet 2016; 29: 547-548

11 Drossman DA. Functional gastrointestinal disorders: history, pathophysiology, clinical features, and Rome IV. Gastroenterology 2016; 150: 1262-1279

12 Edlinger E, Schilling B. Die FODMAP-Ernährungstherapie bei funktionellen Darmerkrankungen. Ernährung und Medizin 2017; 32: 117-118

13 Elsenbruch S, Enck P. Placebo effects and their determinants in gastrointestinal disorders. Nat Rev Gastroenterol Hepatol 2015; 12: 472-485

14 Enck P, Aziz Q, Barbara G et al. Irritable bowel syndrome. Nat Rev Dis Primers 2016; 24: 16014

15 Enck P, Klosterhalfen S, Weimer K. Unsolved, forgotten, and ignored features of the placebo response in medicine. Clin Ther 2017; 39: 458-468

16 Enck P, Mazurak N. Nichtmedikamentöse Therapie des Reizdarmsyndroms. Gastroenterologe 2017; 12: 141-149

17 Eswaran S, Muir J, Chey WD. Fiber and functional gastrointestinal disorders. Am J Gastroenterol 2013; 108: 718-727

18 Federici E, Prete R, Lazzi C et al. Bacterial composition, genotoxicity, and cytotoxicity of fecal samples from individual consuming omnivorous or vegetarian diet. Front Microbiol 2017; 8: 300

19 Ford AC, Talley NJ, Spiegel BM et al. Effect of fibre, antispasmodics, and peppermint oil in the treatment of irritable bowel syndrome: systematic review and meta-analysis. BM] 2008; 337: a2313
20 Francis CY, Whorwell PJ. Bran and irritable bowel syndrome: Time for reappraisal. Lancet 1994; 344: 39-40

21 Frieling T, Krummen, B, Kalde S. Ernährung bei Störung der Verdauungsfunktionen. Ernährung und Medizin 2017; 32: 119-122

22 Frieling T. Reizdarmsyndrom. Falk Foundation M6 1-8/2017PoP

23 Gibson PR, Shepherd SJ. Evidence-based dietary management of functional gastrointestinal symptoms: The FODMAP approach. J Gastroenterol Hepatol 2010; 25: 252-258

24 Haller D, Hörmannsperger G. Darmgesundheit und Mikrobiom. In: Haller D, Grune T, Rimbach G, Hrsg. Biofunktionalität der Lebensmittelinhaltsstoffe. Berlin, Heidelberg: Springer Spektrum; 2013: 67-83, 281-286

25 Halmos EP, Christophersen CT, Bird AR et al. Diets that differ in their FODMAP content alter the colonic luminal microenvironment. Gut 2015; 64: 93-100

26 Halmos EP, Christophersen CT, Bird AR et al. Consistent prebiotic effect on gut microbiota with altered FODMAP intake in patients with Crohn's disease: A randomised, controlled cross-over trial of well-defined diets. Clin Transl Gastroenterol 2016; 7: e164

27 Houghton LA, Heitkemper M, Crowell M et al. Age, gender and women's health and the patient. Gastroenterology 2016; 150; 1332-1343

28 Keefer L, Drossman DA, Guthrie E et al. Centrally mediated disorders of gastrointestinal pain. Gastroenterology 2016; 150: 1408-1419

29 Layer P, Andresen V, Pehl C et al. Irritable bowel syndrome: German consensus guidelines on definition, pathophysiology and management. Z Gastroenterol 2011; 49: 237-293

30 Lebwohl B, Cao Y, Zong G et al. Long term gluten consumption in adults without celiac disease and risk of coronary heart disease: prospective cohort study. BMJ 2017; 357: 1892

31 Lewis JD, Abreu MT. Diet as a trigger or therapy for inflammatory bowel diseases. Gastroenterology 2017; 152: 398-414

32 Lovell RM, Ford AC. Global prevalence of and risk factors for irritable bowel syndrome: a meta-analysis. Clin Gastroenterol Hepatol 2012; 10: 712-721

33 Malfertheiner P. Was ist eine gute Mikrobiota? DGVS 15.9.2017

34 Major G, Pritchard S, Murray K. Colon hypersensitivity to distension, rather than excessive gas production produces carbohydrate-related symptoms in individuals with irritable bowel syndrome. Gastroenterology 2017; 152: 124-133

35 Marsh A, Eslick EM, Eslick GD. Does a diet low in FODMAPs reduce symptoms associated with functional gastrointestinal disorders? A comprehensive systematic review and meta-analysis. Eur J Nutr 2016; 55: 897-906
36 Mazurak N, Broelz E, Storr M, Enck P. Probiotic therapy of the irritable bowel syndrome: Why is the evidence still poor and what can be done about it? J Neurogastroenterol Motil 2015; 21: 471-485

37 Pedersen N, Andersen NN, Vegh Z et al. Ehealth: Low FODMAP diet vs Lactobacillus rhamnosus GG in irritable bowel syndrome. World J Gastroenterol 2014; 20: 1621516226

38 Peters SL, Yao CK, Philpott $\mathrm{H}$ et al. Randomised clinical trial: The efficacy of gut-directed hypnotherapy is similar to that of the low FODMAP diet for the treatment of irritable bowel syndrome. Aliment Pharmacol Ther 2016; 44: 447-459

39 Salazar N, Dewulf EM, Neyrinck AM et al. Inulin-type fructans modulate intestinal Bifidobacterium species populations and decrease fecal short-chain fatty acids in obese women. Clin Nutr 2015; 34: 501-507

40 Schäfer $C$. Food intolerances caused by enzyme defects and carbohydrate malassimiliations: Lactose intolerance and $\mathrm{Co}$. Bundesgesundheitsblatt-Gesundheitsforschung-Gesundheitsschutz 2016; 59: 764-770

41 Schäfer C. Reizender Darm. Hilfestellungen für den Beratungsalltag. Ernährung im Fokus 2010; 9: 410-413

42 Schemann M. Reizdarm und Reizmagen - Pathophysiologie und Biomarker. Der Gastroenterologe 2017; 12: 114-129

43 Schmulson MJ, Drossman DA. What is new in Rome IV. J Neurogastroenterol Motil 2017; 23: $151-163$

44 Schulze-Lohmann P. Ballaststoffe. ErnährungsUmschau 2012; 59: 408-417

Literatur 45-48 ist in der Online-Version unter www.thieme-connect.de/products verfügbar.

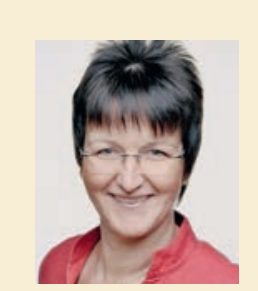

Dipl.oec.troph. Christiane Schäfer Allergologische Schwerpunktpraxis, Team Ernährung Colonnaden 72 20354 Hamburg

Christiane Schäfer ist selbstständige Diplom-Oecotrophologin mit pädagogischem Begleitstudium. Ihr Arbeitsschwerpunkt ist die Allergologie und Gastroenterologie. Neben der Patientenarbeit in einer allergologischen Schwerpunktpraxis ist sie als anerkannte Fachreferentin tätig und Autorin mehrerer Bücher zum Thema Nahrungsmittelunverträglichkeiten.

www.christianeschaefer.de 
45 Shen NT. Timely use of probiotics in hospitalized adults prevents Clostridium difficile infection: a systematic review with meta-regression analysis. Gastroenterology 2017; 152: 1889-1900

46 Shepherd SJ, Parker FC, Muir JG, Gibson PR. Dietary triggers of abdominal symptoms in patients with irritable bowel syndrome: Randomized placebo-controlled evidence. Clin Gastroenterol Hepatol 2008; 6: 765-771

47 Stanghellini V, Chan FK, Hasler WL et al. Gastroduodenal disorders. Gastroenterology 2016; 150: 1380-1392

48 Van Oudenhove L, Crowell MD, Drossmann DA et al. Biopsychosocial aspects of functional gastrointestinal disorders: how central and environmental processes contribute to the development and expression of functional gastrointestinal disorders. Gastroenterology 2016; 150: 1355-1367 